\title{
Successful treatment of solitary intraosseous haemangioma of the femoral neck
}

Zhan $\underline{X i a}^{1}$, MBBS, Kesavan Sittampalam ${ }^{2}$, MBBS, FRCPA, Tet Sen $\underline{\text { Howe }}{ }^{1}$, MBBS, FRCS, Ngai Nung $\underline{L O}^{1}$, MBBS, FRCS

\begin{abstract}
Intraosseous haemangiomas ( $\mathrm{IOHs})$ are benign vascular bone tumours that account for $1 \%$ of all primary bone tumours. They are most frequently seen in the vertebrae and skull, and are rarely found in long bones. Herein, we present an uncommon case of a 25 -year-old woman with a solitary $1 \mathrm{OH}$ that occupied the left femoral neck. We describe the clinical, radiological and histological details of the case, as well as the three-year outcome of the surgical treatment, which successfully preserved the femoral head. We also conducted a review of the literature on this uncommon entity.
\end{abstract}

Keywords: long bone, preservation of femoral head, proximal femur, solitary intraosseous haemangioma, surgical treatment

\section{INTRODUCTION}

Intraosseous haemangiomas $(\mathrm{IOH})$ are benign bone tumours that arise from blood vessels; they account for $1 \%$ of all primary bone tumours. ${ }^{(1)} \mathrm{IOH}$ is one of the few primary bone tumours that has female predominance, with a female-to-male ratio of $3: 2 .^{(2)}$ It is most frequently seen in the vertebrae $(30 \%-50 \%)$ and skull $(20 \%)$, while involvement of the appendicular skeleton, such as long tubular bones, is less frequent. ${ }^{(3)}$ Solitary $\mathrm{IOH}$ in long bones is usually located in the diaphyseal and metadiaphyseal regions. ${ }^{(2)}$ Herein, we present an uncommon case of a large, solitary $\mathrm{IOH}$ in the femoral neck. We report the clinical, radiological and histological details of the case, as well as the three-year outcome of the surgical treatment, which aimed to preserve the femoral head.

\section{CASE REPORT}

A 25-year-old woman presented with left groin pain that persisted for two months. She had no history of trauma or fever, and no history of any remarkable medical or surgical illnesses. On physical examination, there was left groin tenderness and the motion of her left hip joint was limited due to pain. She walked with an antalgic gait and the hip pain intensified with weightbearing activities. Imaging was undertaken, including plain radiography (Figs. 1a \& b), computed tomography (CT) (Fig. 2), magnetic resonance (MR) imaging (Fig. 3) and Technetium-99m methylene diphosphonate (Tc-99m MDP) bone scintigraphy. The radiologic and scintigraphic findings obtained were consistent with that of a solitary $\mathrm{IOH}$, although other diagnostic possibilities (e.g. fibrous dysplasia and aneurysmal bone cyst) had to be considered. In view of the patient's symptoms, and the size and site of the lesion, the patient was deemed to be at risk of a femoral neck fracture. Surgical treatment to remove the lesion and reinforce the weakened femoral neck was recommended, as a fractured femoral neck in a 25-year-old woman could cause avascular necrosis of the femoral head, which would require early prosthetic surgery.

Prior to surgery, we carried out an angiography to explore the possibility of embolisation. However, the angiogram revealed a cavernous haemangioma with no multiple arterial feeders; there was no suitable artery to be considered for embolisation (Fig. 4).
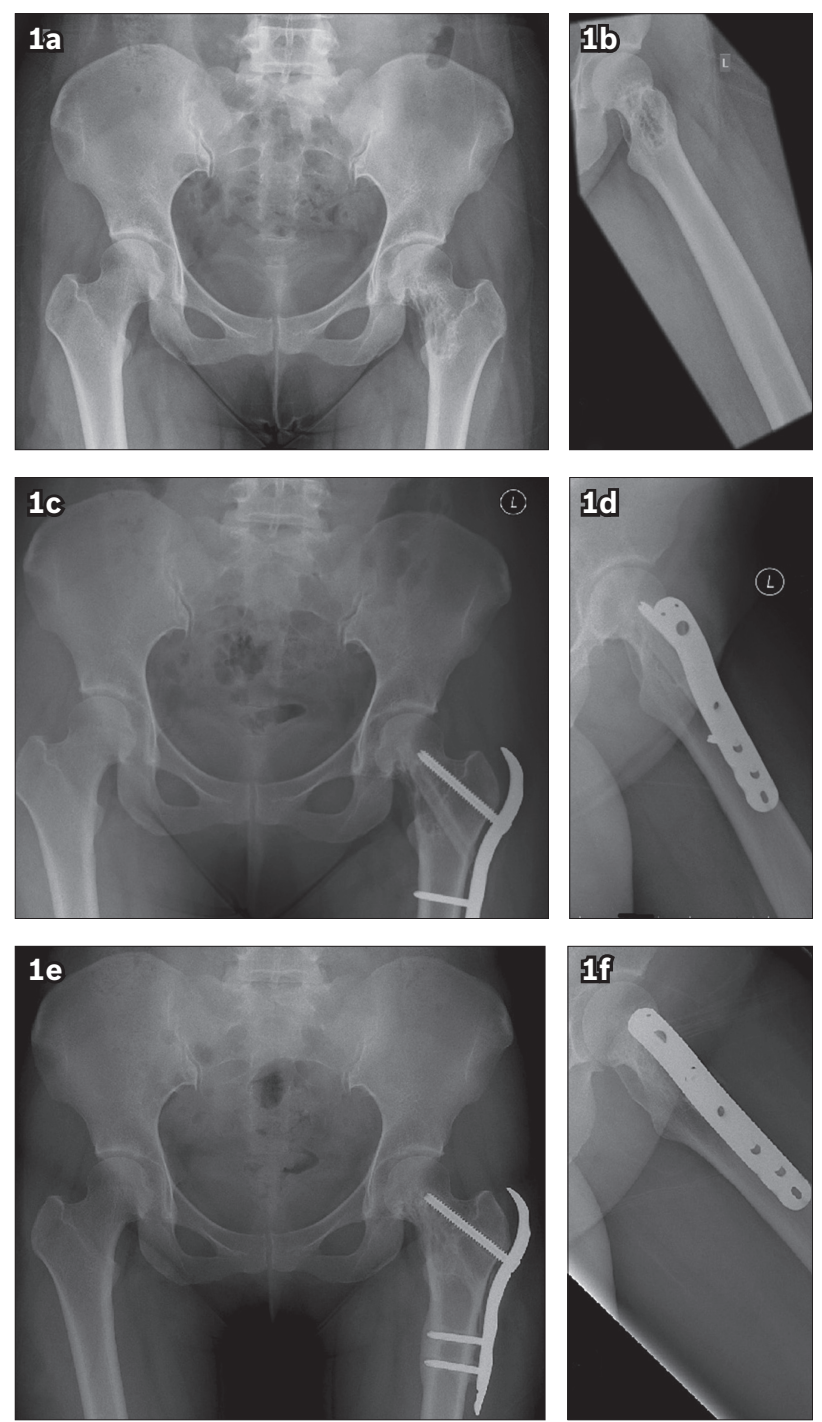

Fig. 1 (a) Anteroposterior radiograph of the pelvis and (b) lateral radiograph of the left hip show a multilocular lucent lesion over the left femoral neck. The lesion extends to the femoral head and intertrochanteric area, with sclerotic borders and internal trabeculation. Three-week postoperative (c) anteroposterior radiograph of the pelvis and (d) lateral radiograph of the left hip show that the lesion was curetted and an allogenic fibular cortical strut, with proximal femoral locking plate and screws in situ, was applied. (e \& f) Repeat radiographs taken during follow-up at 39 months show no recurrence and a well-incorporated allograph. 

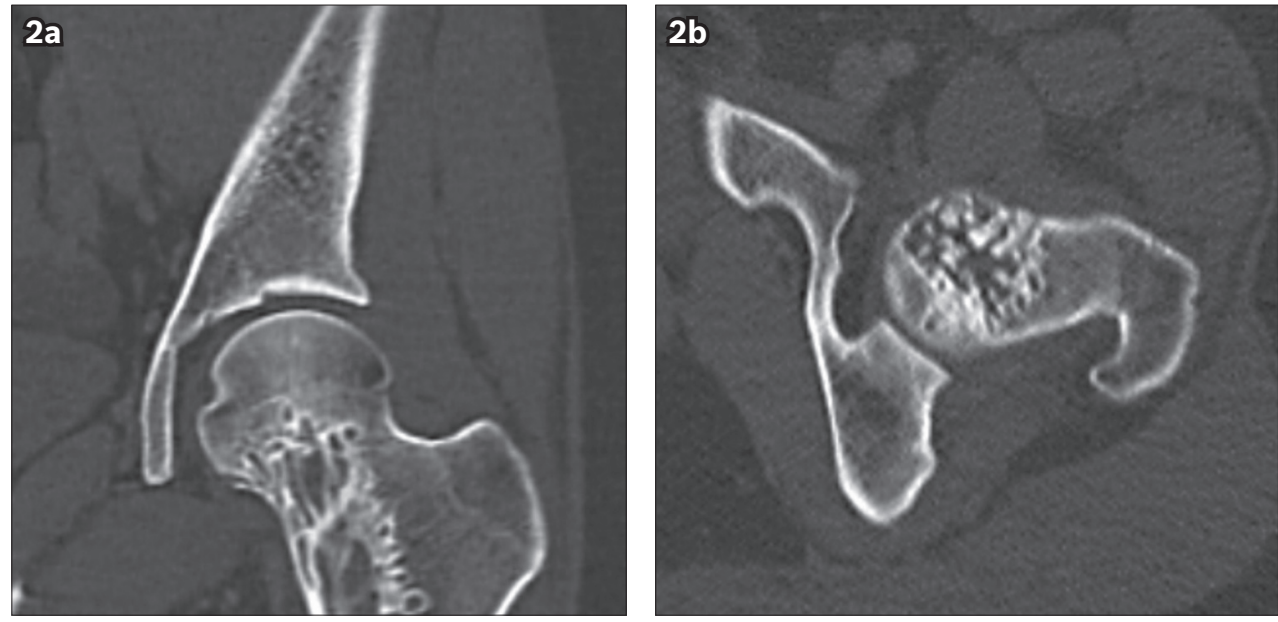

Fig. 2 (a) Coronal CT image of the left hip shows a lesion occupying the medial aspect of the left femoral head and neck. The lesion has an irregular margin, a narrow transition zone and coarse trabeculation within. (b) Axial CT image shows multiple 'dots' within the lesion.
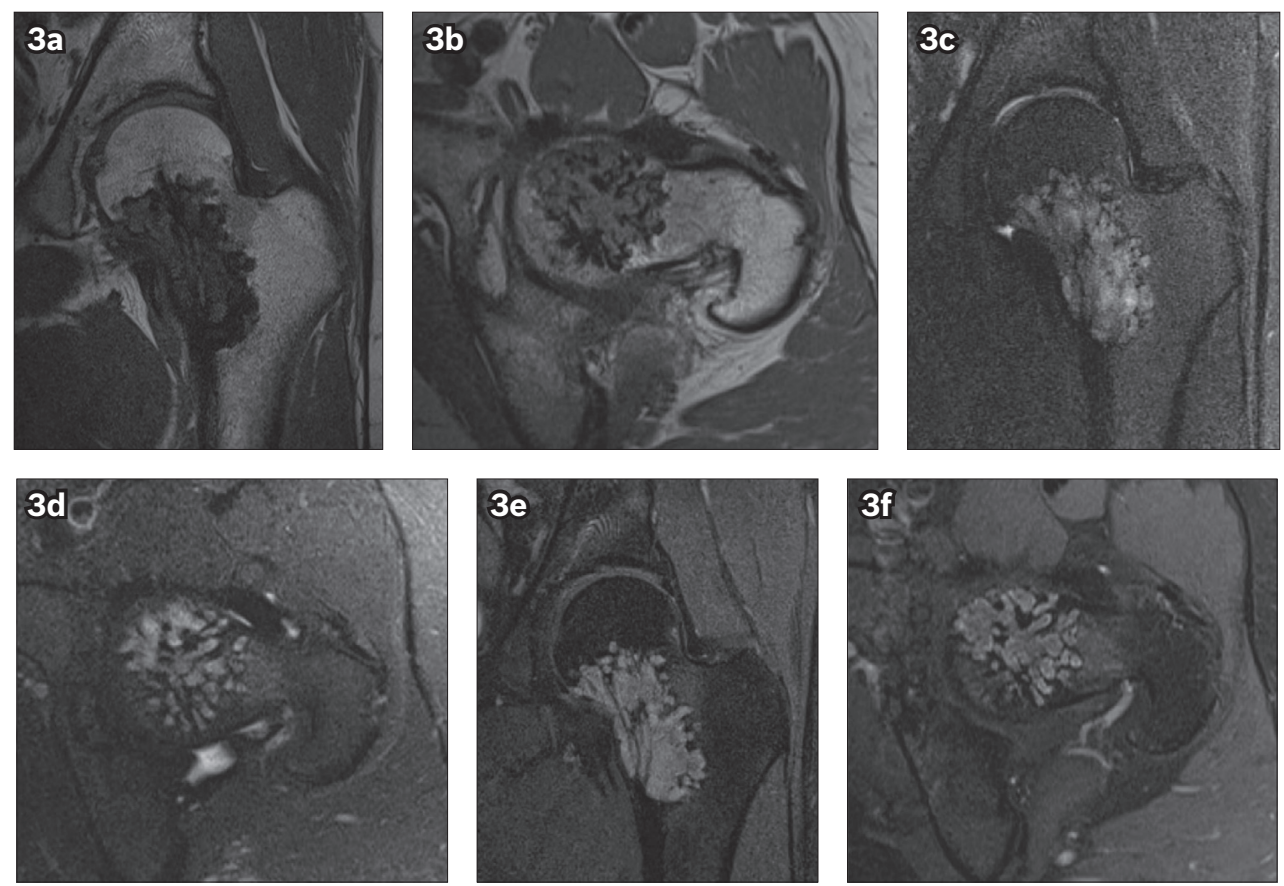

Fig. 3 MR images of the left hip show a $5.7 \mathrm{~cm}$ (craniocaudal) $\times 2.7 \mathrm{~cm}$ (mediolateral) $\times 3.6 \mathrm{~cm}$ (anteroposterior) irregularly marginated lesion with a narrow zone of transition. The lesion occupies the medial aspect of the left femoral neck, and extends into the femoral head and upper metaphyseal region. Thickened trabeculae is observed within the lesion. The lesion is of low signal intensity on ( $\mathrm{a} \& \mathrm{~b}$ ) T1-W images, and of high signal intensity on (c \& d) T2-W images. The spaces between the trabeculae within the lesion show profound enhancement on (e \& f) post-contrast images.

We subsequently performed curettage of the $\mathrm{IOH}$ at the left femoral neck. This was done under a general anaesthetic, with the patient on a traction table to facilitate $\mathrm{C}$-arm imaging. The lesion was located and a guidewire was placed under $\mathrm{C}$-arm guidance. An 11-mm cannulated reamer was passed over the guidewire to create a core from the lateral femoral cortex, along the inferior aspect of the femoral neck and onto the lesion. Long, curved spinal curettes were passed through this channel to excoriate the $\mathrm{IOH}$ (Fig. 5a). No major bleeding was encountered at this stage. A freeze-dried allograft fibular strut was then inserted snuggly into the 11-mm channel (Fig. 5b). Finally, a proximal femoral locking plate was applied with one locking screw inserted through the superior aspect of the femoral neck. This served to load-share and protect against stress fracture of the femoral neck during the incorporation of the allograft fibular. The estimated amount of blood lost during the surgery was $200 \mathrm{~mL}$; perioperative blood transfusion was not required. Histological examination showed that the lesion had features that were consistent with that of a cavernous haemangioma (Fig. 6). Based on the clinical, radiographic, angiographic and histological findings, the diagnosis of cavernous $\mathrm{IOH}$ was constituted.

Postoperatively, repeat plain radiography was conducted (Figs. 1c \& d), and the patient was allowed to ambulate with a 'touch-down' gait (a weight-bearing status in which the foot touches the floor to maintain balance but does not support any weight) for six weeks. Thereafter, she gradually progressed to a partial 

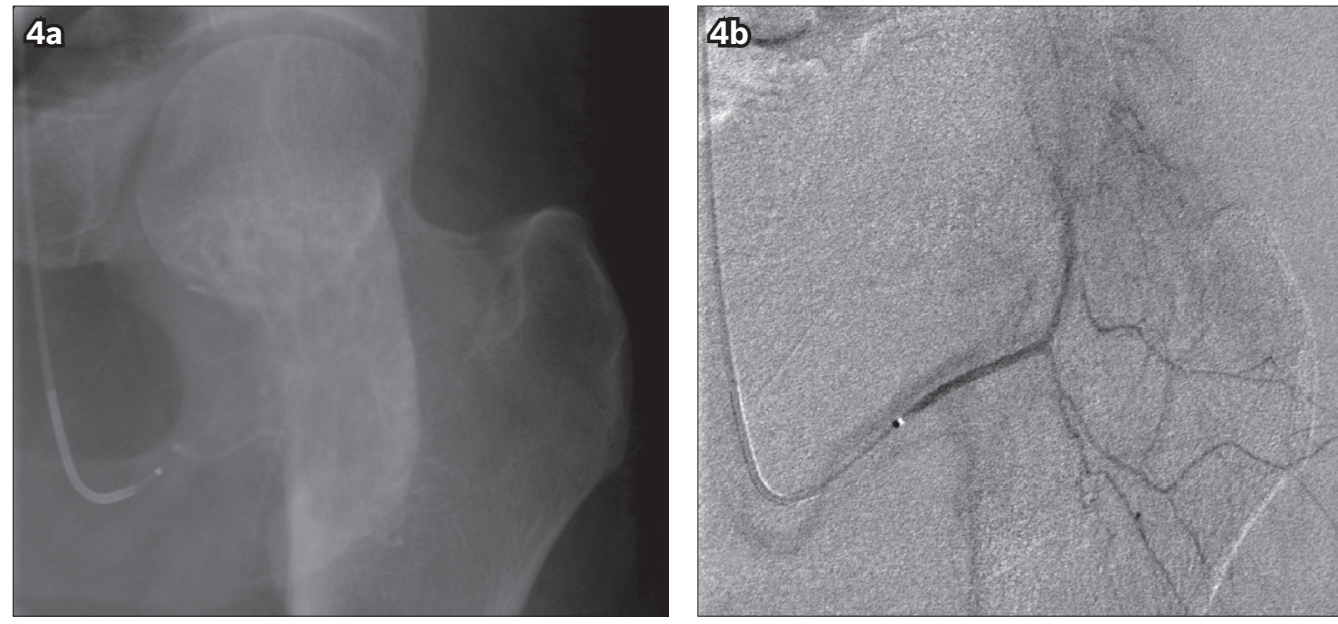

Fig. 4 (a) Radiograph and (b) angiogram of the left hip with selective injections of arteries supplying the hip region show no multiple arterial feeders to the lesion. No arteries were identified to be appropriate for embolisation.
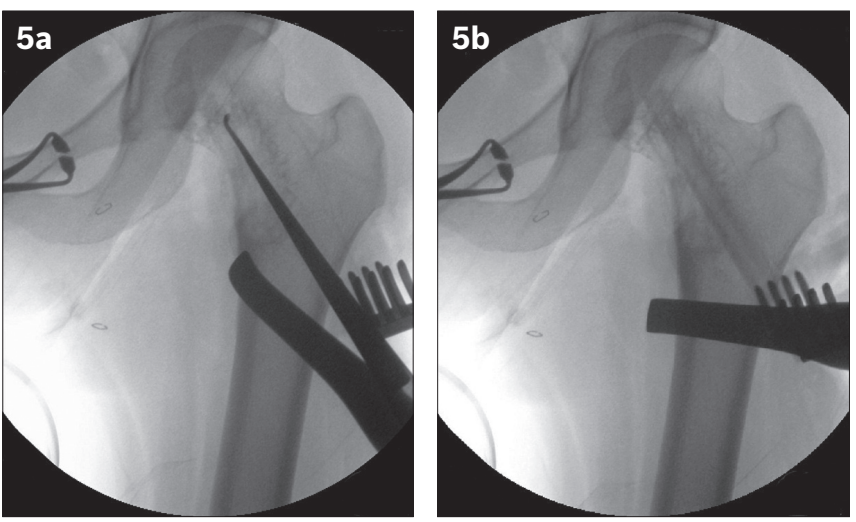

Fig. 5 Intraoperative fluoroscopic images show (a) the lesion being curetted with a long handle curette, and (b) a freeze-dried allograft fibular cortical strut being inserted into the channel, which was created by a $11-\mathrm{mm}$ cannulated reamer.

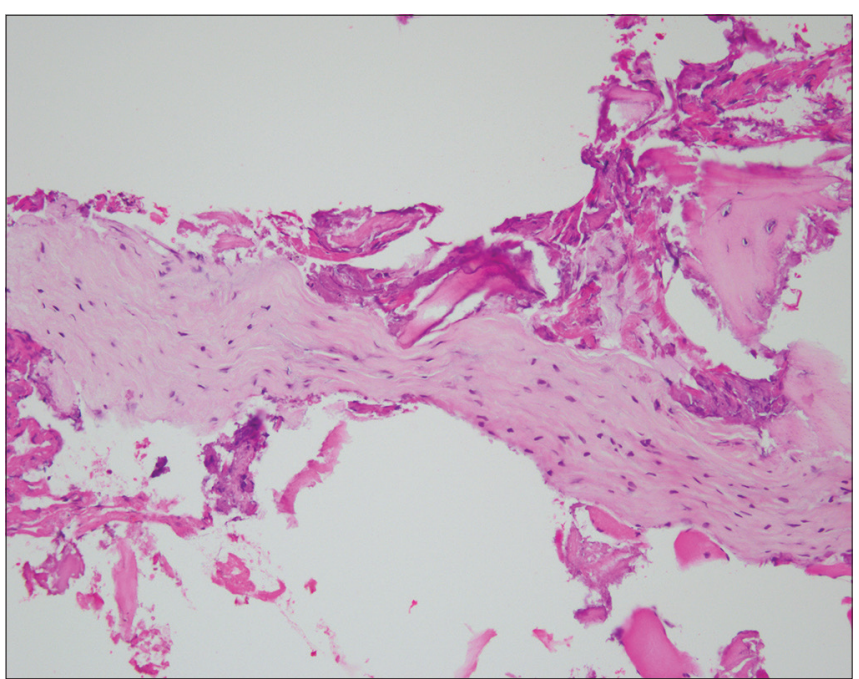

Fig. 6 Photomicrograph shows elongated strips of cellular fibroblastic tissue, consistent with septae from a haemangioma. No endothelial lining or malignancy is identified. These features are consistent with a cavernous haemangioma (Haematoxylin \& eosin, × 200).

weight-bearing status, and at 12 weeks after surgery, she was at full weight-bearing status. She recovered well with full range of painless hip motion and was able to return to her job as an office manager. There were no postoperative complications. Radiographs, which were taken during follow-up at 39 months, showed incorporation of the implanted fibula strut allograft and no recurrence of the lesion or avascular necrosis of the femoral head (Figs. 1e \& f).

\section{DISCUSSION}

$\mathrm{IOH}$ was first described in the literature by Toynbee in $1845 .^{(4)}$ Hitzrot reported the first complete and illustrated case of a solitary $\mathrm{IOH}$ in an extremity in 1917.(5) As mentioned in the study by Kaleem et al, it is extremely rare for a radiologist to encounter a primary haemangioma in the long bones. ${ }^{(2)}$ The rarity of $\mathrm{IOH}$ in the extremities is often emphasised. In fact, we were only able to find two other articles on solitary $\mathrm{IOH}$ in the proximal femur in the literature. This was despite the fact that the proximal femur is one of the most common locations for bony benign lesions. ${ }^{(6)}$ Kaleem et al conducted a literature review of solitary $\mathrm{IOH}$ of the extremities in 2000.(2) In that review, the authors yielded a large group of cases $(n=34)$ in which substantial clinical, radiological and histological details were provided, but none of the cases involved the proximal femur. ${ }^{(2)}$ Chawla et $\mathrm{al}^{(7)}$ reported one case of $\mathrm{IOH}$ involving the femoral head in 2006 and Ferrari et $\mathrm{al}^{(8)}$ described a case of $\mathrm{IOH}$ at the intertrochanteric area of the femur in 2011. The comparison between the previous studies and the present case is summarised in Table I.

$\mathrm{IOHs}$ of the axial skeleton are usually asymptomatic, while those over the appendicular skeleton, depending on the size and site of lesion, are often associated with symptoms such as pain, swelling and pathological fractures.(2) According to the site of the lesion, IOHs of tubular bones can be classified into medullary, periosteal and intracortical IOHs. Medullary $\mathrm{IOH}$ s can be further subcategorised into diaphyseal (48\%), metadiaphyseal (30\%), metaphyseal (12\%), metaepiphyseal (4\%), epimetadiaphyseal (3\%), epiphyseal (1.5\%) IOHs, and $\mathrm{IOH}$ over the total length of the bone $(1.5 \%){ }^{(7)}$ In the present case, the patient presented with groin pain and her $\mathrm{IOH}$ lesion was of the metaepiphyseal medullary type.

The literature describes the radiological patterns of $\mathrm{IOH}$ in the appendicular skeleton as being highly variable and usually nonspecific; a preoperative diagnosis is made only in a minority of cases. ${ }^{(2)}$ On radiography, medullary $\mathrm{IOH}$ may show lytic, 
Table I. Comparison of two cases of previously reported solitary proximal femoral intraosseous haemangioma (IOH) and the present case.

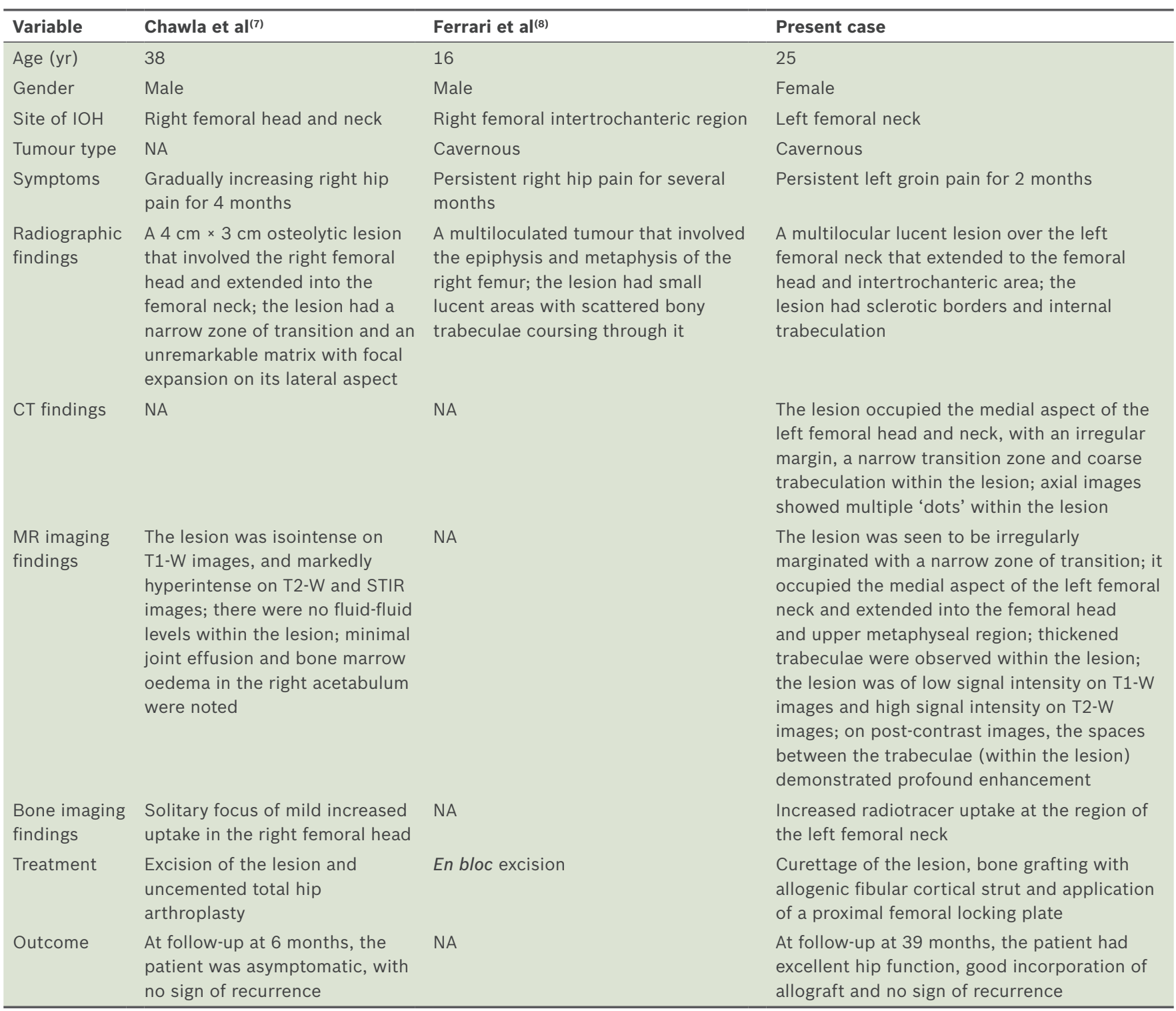

CT: computed tomography, IOH: intraosseous haemangioma; MR: magnetic resonance; NA: not available; STIR: short tau inversion recovery; T1-W: T1-weighted; T2-W: T2-weighted

well-defined, slightly expansile lesions that have a coarse trabecular pattern, which produces a characteristic sunburst, soap bubble/honeycomb appearance due to the expansive proliferation of engorged vessels and thickened, remodelled bone trabeculae. ${ }^{(3,7)}$ Alternatively, it may present with an expansile osteolytic appearance. ${ }^{(9)}$ The appearance of $\mathrm{IOH}$ s on $\mathrm{CT}$ corresponds with its appearance on plain radiography. The cross-sectional images may demonstrate the lesion with a typical 'polka dot' pattern, as the thickened vertical trabeculae are seen as small punctuate areas of sclerosis. ${ }^{(1)}$ MR imaging of the medullary $\mathrm{IOH}$ usually shows variable signal intensity on T1-weighted sequences and high signal intensity on T2-weighted sequences due to water in the stagnant blood within the haemangioma; contrast-enhanced images can show avid enhancement. ${ }^{(1,3)}$ Corresponding to the 'polka dot' pattern observed in axial CT images, axial MR images may also reveal multiple dots.(2) Tc-99m MDP bone scintigraphy is considered nonspecific, with either variable degrees of radiotracer uptake or absence of tracer accumulation. The classical findings of these different diagnostic modalities help contribute to a definite imaging-based diagnosis. This, however, can only be made in a small number of patients with $\mathrm{IOH}$; in patients without pathognomonic radiological features, histology is required for diagnosis. In the present case, our patient's lesion had the typical radiological characteristics of medullary $\mathrm{IOH}$ - a honeycomb appearance on radiographs, a 'polka dot' pattern on axial CT and MR images, high signal intensity on T2-weighted MR images, and marked enhancement on MR images after contrast administration. It was one of those cases in which a definite imaging-based diagnosis of $\mathrm{IOH}$ could be made preoperatively.

Due to the rarity of $\mathrm{IOH}$ and the lack of consistent radiological features, histology plays an important role in the final diagnosis. This is especially so for the examination of en bloc specimens. For the examination of biopsy specimens, curettage or needle biopsy procedures may disrupt the thinwalled blood vessels of the lesion, rendering histological findings 
nonspecific. ${ }^{(1)}$ In such cases, the final diagnosis usually relies on a comprehensive analysis of the clinical, radiological and histological findings. Histologically, $\mathrm{IOH}$ can be classified as cavernous, capillary, venous or mixed, depending on the type of vascular involvement. ${ }^{(2)}$ Among these, cavernous $\mathrm{IOH}$ is the most common type of vascular involvement, accounting for $50 \%$ of all types of vascular involvement, while capillary $\mathrm{IOH}$ accounts for $10 \% .^{(7)}$ In the present case, the diagnosis of cavernous $\mathrm{IOH}$ was made based on radiographic and angiographic findings. The histological features of the biopsy specimens from curettage were consistent with the diagnosis made.

Treatment of benign bone lesions of the proximal femur depends on the size and site of the lesion, as well as the effect that the lesion has on the bony framework. For small, silent lesions that occur without any weakening of the bony framework, close observation can be considered. Some nonoperative treatments specific to $\mathrm{IOH}$ may also be considered; they include direct sclerotherapy, radiotherapy and selective arterial embolisation. ${ }^{(2,10)}$ If the lesion is large and causes weakening of the bony framework (thus rendering impending or actual pathological fracture), surgical treatment is frequently indicated. ${ }^{(6)}$ For lesions involving the articular surface of the femoral head, resection and reconstruction with hip endoprosthesis is often required. ${ }^{(7,11)}$ If the lesion involves the femoral neck or intertrochanteric area with a risk of impending pathological fracture, but preservation of the hip articular surface is possible, curettage and bone grafting with application of an internal fixation device is preferred and has been described with excellent outcomes in previous studies. ${ }^{(6,12,13}$

For bone grafting, cortical bone grafts such as fibular cortical strut grafts are favoured, as they work as a mechanical strut and biological bone graft. The choice between autogenous or allogenous cortical grafts depends on multiple factors. Autogenous cortical grafts are superior to allografts in their rate and completeness of incorporation; this is especially the case for large solitary lesions. ${ }^{(6)}$ The drawbacks include donor site morbidity and limited supply. ${ }^{(14)}$ Allografts, on the other hand, offer the advantages of reduced rate of graft resorption, no donor site morbidity and wide availability; however, they may have a higher rate of infection, a reduced rate of incorporation and a small risk of disease transfer and immunogenicity. ${ }^{(14,15)}$ Augmentation with an internal fixation device can provide additional mechanical support, so as to lessen the risk of a pathological stress fracture, especially during the initial 6-12 months before significant graft incorporation. ${ }^{(13,15)}$ Based on previous studies, ${ }^{(6,12,13)}$ the compression hip screw appears to be the most commonly applied internal fixation device. In the case described by Chawla et al, (7) excision of the femoral head and total hip arthroplasty was unavoidable even though the patient was young, as there was involvement of the articular surface of the femoral head. In the case reported by Ferrari et al, ${ }^{(8)}$ the teenage patient who had $\mathrm{IOH}$ with no direct involvement of the articular surface of the femoral head underwent en bloc resection of the lesion, as the lesion over the intertrochanteric area was considered too large to allow for preservation of the femoral head.

In the surgical treatment of solitary $\mathrm{IOH}$ of the extremities, a common concern is intraoperative bleeding. Yu et $\mathrm{al}^{(10)}$ described a case in which a patient who had an $\mathrm{IOH}$ over the left humerus was initially misdiagnosed with fibrous hyperplasia. In that case, the first attempt at surgery was abandoned when the surgeons encountered uncontrolled haemorrhage during the attempt to curette the lesion. ${ }^{(10)}$ Therefore, in the surgical treatment of an $\mathrm{IOH}$, detailed preoperative plans are essential so as to avoid massive blood loss. Preoperative angiography can be used to identify the major blood vessels that supply the $\mathrm{IOH}$. If the $\mathrm{IOH}$ is supplied by a non-trunk vessel, embolisation should be performed; however, if the blood supply of the $\mathrm{IOH}$ is mainly from a trunk vessel, a technique introduced by Yu et al, in which a balloon was preset to temporarily block the blood flow, ${ }^{(10)}$ could be used. Other considerations may include preoperative preparation of adequate blood products, and perioperative use of recombinant human erythropoietin and iron.

Similar to the case described by Ferrari et $\mathrm{al}^{\left({ }^{(8)}\right.}$ our patient was young and the $\mathrm{IOH}$ did not involve the articular surface of the femoral head. The primary objective in the present case was to remove the $\mathrm{IOH}$, prevent a pathological fracture and preserve the femoral head. Although preoperative embolisation was considered, angiography revealed no major arterial feeders for the $\mathrm{IOH}$, thus rendering embolisation unnecessary. Curettage of the lesion was successfully performed without gross blood loss. Following curettage, we chose to use an allograft fibular cortical strut for our patient, as we were concerned about the possibility of recurrence and an allograft strut is less prone to resorption by the vascular lesion than an autograft strut in recurrence cases. As the lesion involved over $50 \%$ of the femoral neck, we decided to use a proximal locking plate with a 7.3-mm locking screw applied to the superior aspect of the femoral neck. We did not use a compression hip screw with a 12.5-mm lag screw due to concerns that it would be too large for the femoral neck, thus possibly increasing the likelihood of femoral neck fracture during screw placement. The outcome of the surgery in the present case was successful. The patient recovered with excellent hip function, and the allograft fibula strut incorporated well and strengthened the femoral neck. No recurrence was seen during follow-up at 39 months.

\section{REFERENCES}

1. Ching BC, Wong JS, Tan MH, Jara-Lazaro AR. The many faces of intraosseous haemangioma: a diagnostic headache. Singapore Med J 2009; 50:e195-8.

2. Kaleem Z, Kyriakos M, Totty WG. Solitary skeletal hemangioma of the extremities. Skeletal Radiol 2000; 29:502-13.

3. Rigopoulou A, Saifuddin A. Intraosseous hemangioma of the appendicular skeleton: imaging features of 15 cases, and a review of the literature. Skeletal Radiol 2012; 41:1525-36.

4. Toynbee J. An account of two vascular tumors developed in the substance of bones. Lancet 1845; 2:676.

5. Hitzrot JM. Haemangioma cavernosum of bone. Ann Surg 1917; 65:476-82.

6. Shih HN, Cheng CY, Chen YJ, Huang TJ, Hsu RW. Treatment of the femoral neck amd trochanteric benign lesions. Clin Orthop Relat Res 1996; 220-6.

7. Chawla A, Singrakhia M, Maheshwari M, Modi N, Parmar H. Intraosseous haemangioma of the proximal femur: imaging findings. Br J Radiol 2006; 79:e64-6.

8. Ferrari L, Segura Fonseca JJ. Cavernous hemangioma of the proximal femur. Int J Surg Pathol 2011; 19:617-9. 
9. Matsumoto K, Ishizawa M, Okabe H, Taniguchi I. Hemangioma of bone arising in the ulna: imaging findings with emphasis on MR. Skeletal Radio 2000; 29:231-4.

10. Yu L, Cai L, Yu G, Zeng Z, Tao S. Solitary giant hemangioma of the humerus. Orthopedics 2009; 32:925.

11. Zacherl M, Gruber G, Glehr M, et al. Surgery for pathological proximal femoral fractures, excluding femoral head and neck fractures: resection vs. stabilisation. Int Orthop 2011; 35:1537-43.

12. Jaffe KA, Dunham WK. Treatment of benign lesions of the femoral head and neck. Clin Orthop Relat 1990; 134-7.

13. Jaffe KA, Launer EP, Scholl BM. Use of a fibular allograft strut in the treatment of benign lesions of the proximal femur. Am J Orthop (Belle Mead NJ) 2002; 10:575-8.

14. Bryant DD, 3rd, Grant RE, Tang D. Fibular strut grafting for fibrous dysplasia of the femoral neck. J Natl Med Assoc 1992; 84:893-7.

15. George B, Abudu A, Grimer RJ, Carter SR, Tillman RM. The treatment of benign lesions of the proximal femur with non-vascularised autologous fibular strut grafts. J Bone Joint Surg Br 2008; 90:648-51. 